\title{
Eine Frage der Kontrolle: Zunehmende Abgabe der Eigenverantwortung für die Gesundheit und was das mit uns zu tun hat
}

\author{
Holger Cramer \\ Klinik für Naturheilkunde und Integrative Medizin, Evang. Kliniken Essen-Mitte, Medizinische Fakultät, Universität \\ Duisburg-Essen, Essen, Deutschland
}

Seit 2007 ist die Akupunktur in Deutschland Kassenleistung bei Kniearthrose und Rückenschmerzen, 72\% der Orthopäden wenden sie mindestens einmal monatlich an. Hausärzten hingegen haben es eher Phytotherapeutika (90\%), Supplemente (66\%) oder Homöopathika (56\%) angetan [1]. Offenbar hat die Komplementärmedizin das deutsche Gesundheitssystem in den letzten Jahrzehnten tief durchdrungen. Oder etwa doch nicht? Bedeutet komplementär- oder gar integrativmedizinisch $\mathrm{zu}$ arbeiten tatsächlich nur, konventionelle durch unkonventionelle Medikamente oder Kniespiegelungen durch Akupunktur zu ersetzen, weil die Patienten es so verlangen oder die Kassen es refinanzieren? Oder gehört zur Komplementärmedizin nicht noch etwas mehr als das Setzen von Akupunkturnadeln oder das Verschreiben von Kräutertees? So ist die Pflanzenheilkunde ja nur eine Säule der klassischen Naturheilkunde; schon die Kneippsche Wasserkur verlangt vom Patienten viel Eigeninitiative, die Ernährungs-, Bewegungs- und Ordnungstherapie umso mehr. Die moderne Mind-BodyMedizin, die diese traditionellen europäischen Ansätze unter anderem um Meditation oder Yoga ergänzt, ebenso. Und auch die TCM ist ja weit mehr als Akupunktur und Kräutertherapie; Tai Chi und Qigong sind ebenfalls Verfahren, die verlangen, dass der Patient sie selbst umsetzt.

Befragt man komplementärmedizinische Praktiker (wohlgemerkt, keine Ärzte, die auch mal pflanzliche Mittel aufschreiben, sondern hauptberufliche Akupunkteure, TCMler oder Kräuterkundler), so fällt das Urteil eindeutig aus: In der Komplementärmedizin gehe es nicht darum, Patienten mit anderen Mitteln zu heilen, als es die konventionelle Medizin tut. Vielmehr gehe es darum, diesen die Verantwortung für ihre Gesundheit zurück- und ihnen Strategien an die Hand zu geben, sich selbst zu heilen [2].

Die Psychologie spricht hier von Kontrollüberzeugungen: Der Behaviorismus, der die Psychologie und damit unser Verständnis des menschlichen Geistes einen Großteil des letzten Jahrhunderts über geprägt hat, hat menschliches Verhalten weitgehend auf Reiz-Reaktions-Vorgänge reduziert und das eigentliche Studienobjekt der Psychologie, die Psyche halt, als unstudierbar ausgeblendet. Menschliches Verhalten werde, zumindest nach der radikalen Strömung des Behaviorismus, weitgehend durch Belohnung und Bestrafung bedingt: Verhalten, welches positive Folgen nach sich zieht, werde vermehrt, solches mit negativen Folgen seltener ausgeübt. Soweit so einfach, aber leider für die Realität dann doch zu simpel. Denn nicht nur haben Menschen sehr unterschiedliche Einschätzungen, was eine Belohnung und was eine Bestrafung darstellt (man denke nur an Schlagerkonzerte). Auch bedarf es einer klaren Kausalitätszuschreibung, um ein Ereignis, welches auf eigenes Verhalten folgt, als belohnend oder bestrafend zu interpretieren. Viele Menschen schreiben die Folgen eigenen Verhaltens aber eher Glück, Zufall oder "mächtigen Anderen" (Göttern etwa oder Ärzten) zu - und damit auch die Verantwortung für ihr Leben. Daher ändern viele Menschen auch bei negativen Folgen ihr Verhalten 
nicht. Das Konzept der Kontrollüberzeugungen war geboren: Menschen besitzen entweder vorranging internale Kontrollüberzeugungen (sehen also positive oder negative Ereignisse als Konsequenz eigenen Verhaltens) oder externale Kontrollüberzeugungen (sehen das Geschehen also als außerhalb ihrer Kontrolle liegend) [3]. Und dieses Konstrukt der Kontrollüberzeugungen hatte nicht nur für die Psychologie enorme Folgen, indem es dazu beitrug, den Behaviorismus als beherrschende Strömung abzulösen, sondern ist offensichtlich auch für die Komplementärmedizin von überragender Bedeutung:

So ist schon die Konsultation komplementärmedizinischer Anbieter in Deutschland deutlich mit einer hohen internalen gesundheitlichen Kontrollüberzeugung (“Mein Verhalten bestimmt meine Gesundheit!") assoziiert [4]. Und ob Patienten nach einer naturheilkundlichen Behandlung ihr Verhalten ändern, ordnungstherapeutische Empfehlungen also umsetzen oder nicht, wird ebenfalls maßgeblich durch ihre Kontrollüberzeugung mitbestimmt [5]. Die Komplementärmedizin hat und braucht also primär Patienten, die Eigenverantwortung für ihre Gesundheit übernehmen [6]!

Aber davon gibt es in Deutschland immer weniger: Laut eines im letzten Dezember veröffentlichten repräsentativen Surveys der Stiftung Gesundheitswissen glauben nur noch 35 von 100 Menschen, ihre Gesundheit durch ihr eigenes Verhalten stark beeinflussen zu können. Ein Viertel weniger als noch 4 Jahre zuvor! Unter chronisch Kranken sehen 3 von 10 gar keine eigene Einflussmöglichkeit auf ihren Zustand. Auch in der Prävention sieht es düster aus: Weniger als $60 \%$ der Befragten glauben, durch ihr eigenes Verhalten typisch lebensstilassoziierten Krankheiten wie Hypertonie, Typ-II-Diabetes oder auch nur Rückenschmerzen vorbeugen zu können [7]. Komplementärmedizin kommt dabei übrigens besonders schlecht weg: Während $80 \%$ der Befragten der Auffassung sind, ausreichender Schlaf würde ihre Gesundheit fördern, sehen nur 20\% ein solches Potential beim Yoga, gerade einmal 17\% beim Fasten. Und besonders alarmierend ist die Situation bei sozial Benachteiligten: Nicht nur geben diese eine besonders schlechte wahrgenommene Gesundheit und besonders wenige gesundheitsförderliche Verhaltensweisen an. Auch glauben unter Befragten mit niedrigem sozio-ökonomischem Status nur halb so viele wie unter solchen mit hohem Status, dass sie ihren Gesundheitszustand durch ihr eigenes Verhalten stark beeinflussen könnten - nämlich gerade einmal jeder Vierte! Inwieweit sich diese schon niedrigen Zahlen auf Grund der durch die COVID-19-Pandemie zu erwartende Verschärfung der sozialen Ungleichheit [8] noch einmal nach unten korrigieren werden, bleibt abzuwarten.
Droht der Komplementärmedizin in Deutschland also ein Verlust ihrer Grundlage? Werden die vermeintlich einfachen Methoden der Akupunktur, Pflanzenheilkunde oder Homöopathie von der konventionellen Medizin assimiliert und die Methoden der OrdnungstherapieundMind-Body-Medizinverschwinden, da sie schwieriger ins Zeit- und Abrechnungskorsett der Krankenkassen zu pressen sind und von den Patienten eine Eigenleistung voraussetzen, die diese immer weniger bereit sind zu leisten? Ich glaube nicht. Denn auch wenn die Zahl der Menschen mit stark internalen gesundheitlichen Kontrollüberzeugungen sinkt, so gibt es sie dennoch. Und viel wichtiger: Studien zur Nutzung von Komplementärmedizin und internalen Kontrollüberzeugungen zeigen zwar, dass beide Variablen korreliert sind. Sie sagen aber nichts über die Kausalität aus [4]. So ist es naheliegend, dass Menschen, die sich selbst in der Verantwortung für ihre Gesundheit sehen, eher komplementärmedizinische Verfahren verwenden. Aber vielleicht ist es ja auch andersherum: Vielleicht steigert die Verwendung von Komplementärmedizin ja die gesundheitliche Eigenverantwortung. So verstehen ja auch Komplementärmediziner ihre Aufgabe: Als Empowerment, als Vermittlung von Gesundheitskompetenz und -kontrolle [2]. Und tatsächlich zeigen Studien, dass naturheilkundlich-komplementärmedizinische Programme nicht nur die Gesundheit ihrer Patienten verbessern, sondern auch ihre Kontrollüberzeugungen verändern: In Richtung einer stärker internalen Kontrollüberzeugung und Eigenverantwortung [9], die gesundheitsförderliches Verhalten erst ermöglichen [5]. Hier hat die Komplementärmedizin eine wichtige Rolle im Gesundheitssystem, die weit über die eigentliche Behandlung hinausgehen kann und auch in Zukunft nicht an Bedeutung verlieren sollte.

\section{Conflict of Interest Statement}

Der Autor gibt keine Interessenskonflikte an.

\section{Funding Sources}

Dieses Editorial erhielt keine Finanzierung. 


\section{References}

1 Linde K, Alscher A, Friedrichs C, Wagenpfeil S, Karsch-Völk M, Schneider A. Belief in and use of complementary therapies among family physicians, internists and orthopaedists in Germany - cross-sectional survey. Fam Pract. 2015;32(1):62-8.

2 Barrett B, Marchand L, Scheder J, Appelbaum D, Plane MB, Blustein J, et al. What complementary and alternative medicine practitioners say about health and health care. Ann Fam Med. 2004;2(3):253-9.

3 Rotter JB. Generalized expectancies for internal versus external control of reinforcement. Psychol Monogr. 1966;80(1):1-28.
4 Schützler L, Witt CM. Internal health locus of control in users of complementary and alternative medicine: a cross-sectional survey. BMC Complement Altern Med. 2014;14(1): 320

5 Cramer H, Lauche R, Moebus S, Michalsen A, Langhorst J, Dobos G, et al. Predictors of health behavior change after an integrative medicine inpatient program. Int J Behav Med. 2014;21(5):775-83.

6 Walach $\mathrm{H}$. We need a new narrative: agents instead of patients. Complement Med Res. 2018;25(6):364-6.

7 Stiftung Gesundheitswissen. Statussymbol Gesundheit - Wie sich der soziale Status auf Prävention und Gesundheit auswirken kann [aufgerufen 15. Dezember 2020]. Verfügbar unter: https://www.stiftung-gesundheitswissen.de/sites/default/files/pdf/SGW-Gesundheitsbericht_2020_Statussymbol\%20Gesundheit.pdf
8 Kohlrausch B, Zucco A, Hövermann A. Verteilungsbericht 2020 - Die Einkommensungleichheit wird durch die Corona-Krise noch weiter verstärkt. WSI Report 2020;62:1-22 [aufgerufen 15. Dezember 2020]. Verfügbar unter https://www.boeckler.de/pdf/p_wsi_ report_62_2020.pdf

9 Hoffmann B, Moebus S, Michalsen A, Paul A, Spahn G, Dobos GJ, et al. Gesundheitsbezogene Kontrollüberzeugung und Lebensqualität bei chronisch Kranken nach stationärer Behandlung mit Integrativer Medizin - eine Beobachtungsstudie. Forsch Komplementärmed Klass Naturheilkd. 2004;11(3): 159-70. 